\title{
BEDEUTUNG UND AUFBAU VON VERTRAUEN IN DER SOZIALPÄDAGOGISCHEN FAMILIENBEGLEITUNG
}

Cornelia Rüegger, Joel Gautschi, Roland Becker-Lenz und Fabienne Rotzetter

Hochschule für Soziale Arbeit FHNW, Institut für Professionsforschung und -entwicklung

E-Mail: cornelia.rueegger@fhnw.ch

URL: https://www.fhnw.ch/de/personen/cornelia-rueegger

ZHAW Zürcher Hochschule für angewandte Wissenschaften

E-Mail: joel.gautschi@zhaw.ch

URL: https://www.zhaw.ch/de/ueber-uns/person/gaud

Hochschule für Soziale Arbeit FHNW, Institut für Professionsforschung und -entwicklung

E-Mail: roland.becker@fhnw.ch

URL: https://www.fhnw.ch/de/personen/roland-becker-lenz

Hochschule für Soziale Arbeit FHNW, Institut für Professionsforschung und -entwicklung

E-Mail: :fabienne.rotzetter@fhnw.ch

URL: https://www.fhnw.ch/de/personen/fabienne-rotzetter

Zitationsvorschlag:

Rüegger, Cornelia/Gautschi, Joel/ Becker-Lenz, Roland/Rotzetter, Fabienne (2021): Bedeutung und Aufbau von Vertrauen in der Sozialpädagogischen Familienbegleitung. In: Gesellschaft - Individuum - Sozialisation (GISo). Zeitschrift für Sozialisationsforschung, 2 (2). DOI: 10.26043/GISo.2021.2.3

Link zum Artikel:

https://doi.org/10.26043/GISo.2021.2.3 


\title{
BEDEUTUNG UND AUFBAU VON VERTRAUEN IN DER SOZIALPÄDAGOGISCHEN FAMILIENBEGLEITUNG
}

\author{
Cornelia Rüegger, Joel Gautschi, Roland Becker-Lenz und \\ Fabienne Rotzetter
}

Der Aufbau von Vertrauen wird in Theorie und Praxis Sozialer Arbeit als Voraussetzung für Hilfe diskutiert. Wenn eine sozialpädagogische Familienbegleitung (SPF) als Massnahme im zivilrechtlichen Kindesschutzbereich angeordnet ist, geht ihr Hilfeauftrag mit einem Schutzauftrag einher, manchmal auch gegen den Willen der Betroffenen. Es ist anzunehmen, dass der Aufbau einer vertrauensvollen Zusammenarbeit erschwert ist. Der Beitrag beleuchtet auf der Grundlage einer rekonstruktiven Studie den Aufbau von Vertrauen im Ausgangspunkt solcher Familienbegleitungen und fokussiert auf vertrauensrelevante Ausgangsbedingungen.

Keywords: Vertrauen, Arbeitsbeziehung, sozialpädagogische Familienbegleitung, Zwangskontext, Kindesschutz

\section{EINLEITUNG}

Für die Sozialisation von Kindern und Jugendlichen nimmt die Familie eine zentrale Bedeutung ein, weil sie ein bedeutender Ort der Bildung der personalen Identität, Integrität und Autonomie eines Menschen ist. Wenn eine Familie im Hinblick auf diese Funktionen defizitär erscheint oder sich selbst in Schwierigkeiten sieht, stehen Angebote der Kinder- und Jugendhilfe wie die Sozialpädagogische Familienbegleitung (SPF) zur Verfügung. Der Fachverband für Sozialpädagogische Familienbegleitung Schweiz definiert ihre Funktion als „aufsuchendes Angebot der Kinderund Jugendhilfe, um Familien bei der Bearbeitung unterschiedlichster familiärer Problemlagen zu unterstützen und dadurch die Lebensbedingungen der betroffenen Kinder und Jugendlichen zu verbessern". ${ }^{1}$ Der Ansatzpunkt dieser Hilfeform ist demnach die Stärkung von Familien als sozialisatorischer Kontext von Kindern und Jugendlichen bei der Bewältigung von Problemlagen. Idealerweise liegt der Ausgangspunkt dieser Unterstützung darin, dass die Eltern einen Bedarf erkennen und im Sinne einer Hilfe zur Selbsthilfe (Oevermann 2009) davon Gebrauch machen. Der Aufbau von Vertrauen und das Einrichten eines Arbeitsbündnis (ebd.) bzw. Vertrauenskontraktes (Schütze 1992) zwischen der Fachkraft der SPF und der Familie gilt gemäss Schütze und Oevermann als Voraussetzung für eine solche Hilfe. Dies umso mehr, da in diesem Setting die Hilfe in der Privatsphäre der Wohnung und im Familienalltag stattfindet. Familiale Problemlagen zeigen sich den Fachkräften hier direkt. Das kann bei den Familienmitgliedern Scham hervorrufen (Gibson 2020) sowie auch Sorgen bezüglich der Beurteilung und Bearbeitung der Probleme durch die Fachkräfte. Die Fachkräfte sind verpflichtet bei Gefährdungen des Kindeswohls die Kindesschutzbehörden zu informieren. Vertrauen kommt hier in Bezug auf die Klientinnen und Klienten die Funktion zu, Sorgen beiseitezuschieben und sich mit positiven Erwartungen auf die SPF einlassen zu können. Ein Erschwernis für die Vertrauensbildung ist zu erwarten, wenn die Hilfe nicht aktiv von den Eltern gesucht, sondern als Massnahme zur Abwendung einer Kindeswohlgefährdung von Behörden angeordnet wird. Studien im Kinderschutz zeigen, wie unter solchen Bedingungen sowohl Fachkräfte als auch Klientinnen und Klienten die Beziehung strategisch gestalten (Klatetzki 2019; Freres 2020). Interventionen, welche von den Eltern als Kontrolle und nicht als Hilfe erlebt werden, können bei diesen zu Aggressionen, aber auch zu Täuschungen (Klatetzki 2019) bzw. einem als „impression management" (Goffman

\footnotetext{
${ }^{1}$ https://www.spf-fachverband.ch/fileadmin/media/downloads/RZ_Flyer_A5_Leitbild_SPF_WEB_mc_230120.pdf
} 
1959) zu verstehendem „playing the game“ auf einer „Vorderbühne“ führen (Dumbrill 2006; Freres 2020). Es ist anzunehmen, dass der Aufbau einer vertrauensvollen Zusammenarbeit in solchen Kontexten erschwert ist, möglicherweise auch nicht gelingt. Gelingt dies nicht, ist fraglich, ob der mit einer ambulanten Kinderschutzmassnahme verbundene Schutzauftrag erfüllt werden kann (Klatetzki 2019).

Der vorliegende Beitrag aus einer qualitativen Studie zu Vertrauen zwischen Klientinnen bzw. Klienten und Fachkräften der Sozialen Arbeit im Kindesschutz richtet den Fokus auf den Aufbau von Vertrauen im Ausgangspunkt solcher Familienbegleitungen und interessiert sich für vertrauensrelevante Ausgangsbedingungen und ihre Bedeutung für den Aufbau von Vertrauen. Dazu wird zunächst im zweiten und dritten Abschnitt auf die Konzeptualisierung von Vertrauen eingegangen und das Forschungsdesign dargestellt. Im vierten Abschnitt werden ausgewählte Ergebnisse zur Vertrauensbildung auf der personalen Ebene vorgestellt. Diese werden im letzten Abschnitt zusammengefasst und diskutiert.

\section{VERTRAUEN ALS BEDEUTSAME KATEGO- RIE IN DER SOZIALEN ARBEIT}

Vertrauen ist ein komplexes Phänomen und erfordert eine konzeptionelle Auseinandersetzung, um diesen Gegenstand fassbar zu machen. Nachfolgend stellen wir dar, wie wir Vertrauen in unserer Studie theoretisch fassen und präsentieren ausgewählte empirische Ergebnisse zu Vertrauen zwischen Fachkräften der Sozialen Arbeit und ihren Klientinnen und Klienten.

\subsection{Zur Konzeption von Vertrauen}

Trotz unterschiedlicher Konzeptionen von Vertrauen hat sich bezüglich wesentlicher Aspekte von Vertrauen über verschiedene Disziplinen hinweg ein Konsens herauskristallisiert (McLeod 2015). Am besten ausgearbeitet ist dies für das "dominante Paradigma" (McLeod 2015) des interpersonalen Vertrauens, welches im Zentrum des vorliegenden Artikels steht. Vertrauen kann als Form von positiven Erwartungen an die Handlung eines anderen Akteurs bzw. einer anderen Akteurin verstanden werden (Möllering 2005, 287). Konstitutiv für Vertrauen ist (Baier
1991; McLeod 2015), dass erstens die vertrauende Person sich verletzlich macht oder verletzlich ist hinsichtlich der Konsequenzen eines Vertrauensbruchs und damit bereit ist, ein gewisses Risiko einzugehen. Zweitens muss die vertrauende Person optimistisch sein, dass die Person, in die sie das Vertrauen setzt, über die für die Erfüllung des Vertrauens notwendigen Fähigkeiten verfügt; und drittens muss sie optimistisch sein, dass das Gegenüber (selbstIverpflichtet bzw. motiviert ist, die in sie gesetzten Erwartungen zu erfüllen.

Mit unserer Vertrauenskonzeption schliessen wir uns Möllering (2006, 105-126) an, wonach sich Vertrauen zwar auf "guter Gründe" oder (auch unbewusste) Routinen stützen kann, sich Vertrauen aber nicht kalkuliert-rational oder automatisch aus diesen ergibt. Damit eine potenziell vertrauensgebende Person von der Interpretation, „gute Gründe zu haben“, zu Vertrauen gelangt, ist vielmehr ein „leap of faith“ bzw. „leap of trust" erforderlich, was als Aufhebung (suspension) von nicht-kalkulierbarer Ungewissheit verstanden werden kann ebd.).

Es würden relevante Phänomenbereiche aus dem Blick geraten, würde Vertrauen und der dafür notwendige „leap of faith“ bloss als innerpsychischer Prozess gefasst (vgl. für diese Position bspw. Hardin 2002, 58-60). Vielmehr ist Vertrauen auch als soziales Phänomen zu fassen (Lewis/Weigert 1985a). So lassen sich kognitive, emotionale und sozial-behaviorale Komponenten von Vertrauen unterscheiden (Lewis/Weigert 1985b). Vertrauen ist nicht nur als Zustand (trust), sondern auch als Prozess (trusting) zu fassen. Vertrauensprozesse müssen in ihrer zeitlichen Entwicklung, deren mentalen und sozialen Dynamiken, dem Zusammenspiel dieser Prozesse und der Auswirkung von Vertrauen auf Personen und soziale Strukturen gedacht werden (Möllering 2013). Dies erlaubt es auch, die strukturellen Bedingungen des in unserer Untersuchung im Fokus stehenden Zwangskontextes und den damit einhergehenden Asymmetrien zwischen Fachkräften und Klientinnen bzw. Klienten mit in den Blick zu nehmen, die sich abhängig von der Position der Beteiligten auf die Vertrauensbildung unterschiedlich auswirken können (allgemein dazu bspw. Baier 1986, 240 244; Cook et al. 2005, 42-47). 
Vom interpersonalen Vertrauen, das bis hier im Zentrum der Ausführungen stand, lässt sich anaIytisch das Vertrauen in abstrakte Systeme bzw. Institutionen unterscheiden (Luhmann 1968; Giddens 1990). Wobei das Vertrauen von Personen in abstrakte Systeme zunächst die Form von gesichtsunabhängigen Bindungen (faceless commitments) hat (Giddens 1990, 80-88). Die vertrauensgebende Personen, wie im Fall der Klientinnen und Klienten der SPF, können jedoch auf Repräsentanten und Repräsentantinnen von abstrakten Systemen treffen, wodurch die Systeme dann nicht mehr nur abstrakt erscheinen (ebd.). Treffen Klientinnen bzw. Klienten auf Fachkräfte, ist analytisch weiter zwischen einem spezifischen Vertrauen der Klientin bzw. des Klienten in die Fachkraft als Rollenträger oder als Rollenträgerin und persönlichem Vertrauen, das sich auf der Grundlage persönlicher Eigenschaften und individueller Verhaltensweisen der Fachkraft entwickelt, zu unterscheiden.

\subsection{Empirische Ergebnisse zu Vertrauen zwi- schen Fachkräften und Klientinnen bzw. Klienten in der Sozialen Arbeit}

In den wenigen verfügbaren empirischen Studien wird Vertrauen in eine gelingende Arbeitsbeziehung in der Sozialen Arbeit eine hohe Bedeutung zugeschrieben (bspw. Fröhlich-Gildhoff 2014; Cossar et al. 2016; Hansjürgens 2018; Husby et al. 2018). Umstritten ist aber, inwieweit auch die oben erwähnten Elemente aus diffusen Sozialbeziehungen in Arbeitsbeziehungen aus Sicht der Adressatinnen und Adressaten bedeutsam sind (verneinend: Wagenblass 2004; Arnold 2009; bejahend: Zeller 2012).

Der Aufbau von Vertrauen kann sowohl bei Fachkräften als auch bei Klientinnen und Klienten durch biographische Erfahrungen, Erfahrungen von relevanten Anderen (Eltern, Peers etc.) und öffentliche Diskurse erschwert oder erleichtert werden (exemplarisch Aspekte davon bei Cleppien 2012; Davies 2019). Ausgangsbedingungen wie generalisiertes Vertrauen in Personengruppen erweisen sich dabei als bedeutsam, wenn auch nicht determinierend, für konkrete Arbeitsbeziehungen.

Damit sich Vertrauen etablieren kann, erfordert dies von den Beteiligten „Vertrauensarbeit“" (Hall et al. 2013). Diese kann sich auch auf die Bearbeitung von ungünstigen Ausgangsbedingungen für Vertrauen beziehen, wie bspw. strukturelles Misstrauen, welches Eltern im Zwangskontext durch „Verdachtsarbeit" in der Abklärung (Franzheld 2017) und daran anschliessenden behördlichen Massnahmen entgegengebracht wird (Brauchli 2021). Studien verweisen auf Praktiken der Fachkräfte, die (zumindest der Intention nach) vertrauensfördernd sind. Die Praktiken lassen sich analytisch den Lefevre et al. (2017) angelehnten, nicht ganz trennscharfen Bereichen zuordnen (Arnold 2009; Tiefel 2012; Hall et al. 2013; Richter 2013; Lefevre et al. 2017; Brauchli 2021; Rüegger 2021): beziehungsorientierte Praktiken (bspw. reziproke Beziehungsgestaltung, Wertschätzung, Interesse zeigen, Vertrauen in die Fähigkeiten der Klientel zeigen, Vermeidung achtungsbedrohender Mitteilungsformen), klientenzentrierte Praktiken (Partizipation, Ausrichtung an den Interessen und Relevanz der Klientel, Interventionen werden als Hilfe zur Erweiterung von Möglichkeiten und nicht als Eingriffshandeln dargestellt) und ethische Praktiken (Nachvollziehbarkeit, Transparenz, Vertraulichkeit) sowie Praktiken bezüglich aufgabenbezogener Fähigkeiten und Wissen (bspw. Kompetenzpräsentation, Zuständigkeit zeigen). Eine besondere Wichtigkeit schreibt Arnold (2009) der Nachvollziehbarkeit des Handelns zu, welches bei der Klientel Orientierung und Sicherheit schafft und damit Vertrautheit als bedeutsame Grundlage für Vertrauen erzeugt.

Die Wechselseitigkeit von Vertrauen zeigt sich empirisch bspw. an der (möglicherweise kontrafaktischen) Vertrauensunterstellung, die Fachkräfte gegenüber Eltern zeigen, indem sie diese als engagiert, verlässlich und kompetent darstellen, und Risiken im Zusammenhang mit elterlichem Handeln, die abgeschwächt und als mitunter extern verursacht dargestellt werden, um dadurch ihr Vertrauen zu gewinnen und die Arbeitsbeziehung aufrechtzuerhalten (Hall et al. 2013; Rüegger 2021). Auf die Bedeutung der Ausrichtung an elterlichen Sichtweisen und Erfahrungen für den Vertrauensaufbau und die damit verbundenen Spannungsfelder verweist Brauchli (2021, 353-361) in ihren Analysen von Gesprächen in der SPF (für die Suchthilfe 
Hansjürgens 2018). Elternteile stellen die Loyalität der Fachkräfte innen gegenüber auf die Probe, um vom darauf bezogenen Handeln der Fachkräfte auf deren Vertrauenswürdigkeit zu schliessen. Dadurch kann es bei den Fachkräften zu Spannungsfeldern kommen zwischen einer vertrauensförderlichen, konsequenten Orientierung an den elterlichen Interessen und der Arbeit an aus Sicht der Fachkraft relevanten, von den Eltern aber nicht als solche anerkannten, Problemen. Brauchli $(2021,360)$ zeigt, wie auch in solchen Spannungsfeldern bei mangelnder Problemeinsicht der Eltern eine Verschiebung der elterlichen Problemperspektive angestossen werden kann, wenn es den Fachkräften gelingt, Problemlösungsvorschläge zu entwickeln, die anschlussfähig daran sind, was die Eltern als Probleme erfahren.

Die vorliegende Studie schliesst an diesen empirischen Ergebnissen an. Der Schwerpunkt des Erkenntnisinteresses liegt bei der noch wenig ausgeleuchteten Bedeutung der unterschiedlichen Ausgangsbedingungen der Fallarbeit in der SPF im Zwangskontext für die Vertrauensarbeit zwischen Fachkräften und ihrer Klientel.

\section{FORSCHUNGSDESIGN}

Für den Beitrag werden Ergebnisse aus dem vom Schweizerischen Nationalfonds geförderten Forschungsprojekt zu Vertrauen zwischen Klientinnen bzw. Klienten und Fachkräften der Sozialen Arbeit im Kindesschutz genutzt. Das Erkenntnisinteresse der Studie richtet sich darauf, 1) welche Bedeutung Vertrauen bzw. der wechselseitigen Vertrauensbildung zwischen Klientinnen bzw. Klienten und Fachkräften in angeordneten sozialpädagogischen Familienbegleitungen im Zwangskontext zukommt, 2) welche Formen von Vertrauen von Relevanz sind und 3) welche Bedingungen und Faktoren für Vertrauen förderlich bzw. hinderlich sind. Geleitet durch die Annahme, dass sich die Arbeitsbeziehung in den ersten Gesprächen der SPF mit der Familie (vorläufig) konstituiert und Vertrauensbildung dabei eine wichtige Rolle spielen könnte, richtete sich der Fokus der Studie auf diese erste Phase der Zusammenarbeit. Um die zeitliche Entwicklung von Vertrauen (trusting als Prozess) beobachten zu können, wurden ein bis zwei Gespräche zu Beginn der Fallarbeit und ein weiteres Gespräch nach zweieinhalb bis sieben Monaten, je nachdem, wann sich eine erste, vorläufige Fallkonstitution (trust als Zustand) beobachten liess, aufgezeichnet (Ton). ${ }^{2}$ Diese Aufzeichnungen sollten es ermöglichen, die emotionale und behavoriale Ebene der Vertrauensbildung zu erfassen. Um auf den Fall und die Vertrauensbildung bezogene Deutungen der Beteiligten sowie deren emotionale Befindlichkeit zu erheben (kognitive und emotionale Ebene der Vertrauensbildung), wurden nach den Gesprächen zwischen SPF und Familienmitgliedern zusätzlich separate leitfadengestützte Interviews mit der SPF-Fachkraft und mindestens einem Familienmitglied (siehe Tabelle 1) geführt. Diese Interviews dienten ebenfalls dazu, die Wechselseitigkeit der Vertrauensbildung zu erfassen. Ausserdem wurden Falldokumente wie Akten, Fallberichte etc. erhoben.

Der Zugang zu den Fällen erfolgte über Organisationen des Fachverbandes SPF Schweiz. Insgesamt wurden im Erhebungszeitrum (07.2018 bis 02.2020) Daten von sechs Fällen aus vier Organisationen erhoben (siehe Tabelle 1). Da sich das Erkenntnisinteresse der Studie auf den Zwangskontext bezog, wurden als Kriterium für die Datenerhebung aufzunehmende Fälle festgelegt, in denen die SPF im Rahmen einer durch die Kindes- und Erwachsenenschutzbehörde (KESB) angeordneten zivilrechtlichen Massnahme der Kinderschutzbehörde erfolgte. ${ }^{3}$ Aufgrund von Schwierigkeiten beim Zugang zu

\footnotetext{
${ }^{2}$ Als „vorläufige Fallkonstitution“ wurde in Absprache mit den Fachkräften jener Moment in der Fallarbeit gewählt, in dem die Fachkräfte auf der Basis der bisherigen Zusammenarbeit mit der Familie und des sozialdiagnostischen Prozesses einen ersten Bericht zuhanden der Behörde erstellten und mit der Familie besprachen. Für eine ausführliche Darstellung zu den Prozessen und Praktiken der Fallkonstitution siehe Rüegger (2021).

3 Die Kindes- und Erwachsenenschutzbehörden haben in der Schweiz die Aufgabe, Kinder und Erwachsene, deren Wohlergehen gefährdet erscheint, zu schützen. Sie klären die Gefährdungslage ab und verfügen ggf. über geeignete Massnahmen zu deren Abwendung. Die Durchführung dieser Massnahmen erfolgt in der Regel durch soziale Dienste und Einrichtungen der Gesundheits- und Sozialhilfe.
} 
geeigneten Fällen wurde dieses Kriterium in einem Fall gelockert.

Die Transkripte der Gesprächsaufzeichnungen wurden mit den Verfahren der objektiven Hermeneutik (Oevermann 2000) analysiert. Wir folgen damit Möllering (2001, 416; 2006), der offene, interpretative Analyseverfahren empfiehlt, um die idiosynkratischen Praxen und Feinheiten der Deutungen der am Vertrauensprozess Beteiligten empirisch fassen zu können. Weiteres Datenmaterial (Interviews, Akten) wurde thematisch kodiert. Ausgewählte Stellen, die für die Vertrauensbildung besonders relevant erschienen, wurden mit objektiver Hermeneutik sequenziell analysiert. Dies waren Stellen, in denen entweder die Schwierigkeiten oder auch die für die Vertrauensbildung förderlichen Umstände deutlich wurden, etwa weil die. Klientinnen bzw. Klienten von ihren Erfahrungen mit Kindesschutzbehörden berichteten, weil implizit schwer vereinbare Erwartungen, Zielsetzungen oder Handlungsstrategien deutlich wurden oder weil auf einer emotionalen Ebene wechselseitige Sympathie herauszulesen war.

\section{EMPIRISCHE ERGEBNISSE}

Die Ergebnisdarstellung fokussiert nun auf den Aufbau von Vertrauen im Ausgangspunkt solcher Familienbegleitungen. Die Analysen verweisen auf verschiedene Einstiegskonfigurationen, die sich für den Vertrauensaufbau im Sinne von günstigen oder weniger günstigen Konstellationen als bedeutsam erweisen. Es sind dies die Vorgeschichte des Falles und vertrauensrelevante Vorerfahrungen der Klientinnen und Klienten mit Hilfesystemen (4.1), Anliegen, Interessen und Erwartungen der Klientel bezüglich der Hilfe (4.2), Risikofaktor Kindeswohlgefährdung (4.3) sowie das "Matching“ auf der Beziehungsebene als Schlüsselfaktor für den Vertrauensaufbau (4.4).

\subsection{Zur Bedeutung der Vorgeschichte des Falles und vertrauensrelevanter Vorerfahrungen der Klientel mit Hilfesystemen}

Gerade bei behördlich angeordneten Massnahmen verfügen die Klientinnen und Klienten beim Erstkontakt mit der SPF über vertrauensrelevante Vorerfahrungen aus ihren Interaktionen mit
Behördenmitgliedern, abklärenden Diensten und professionellen Hilfesystemen unterschiedlicher Art (bspw. Schulsozialarbeit, psychologischen Diensten). Im Datenmaterial werden darüber erworbene Einstellungen auf der Ebene von Systemvertrauen als mehr oder weniger vorhandenes Vertrauen in die KESB sowie bezüglich der angeordneten Massnahme sichtbar, die für den Aufbau von Vertrauen relevant sind.

Positive Erfahrungen und Einstellungen der Klientel

Ein Teil der Klientinnen und Klienten, so in Fall 1, Fall 5 und teilweise auch in Fall 2, verfügen über Erfahrungen mit professionellen Hilfen, die sie als hilfreich erlebt haben. Sie sehen der Zusammenarbeit mit der Fachkraft hoffnungsvoll entgegen und berichten von der Erleichterung Hilfe zu bekommen. In den Erstgesprächen präsentieren sie sich gegenüber den Sozialarbeitenden als offen, motiviert und kooperationsbereit.

Bei diesen positiven Erfahrungen mit professionellen Hilfen und entsprechendem Systemvertrauen handelt es sich zunächst um eine sehr günstige Einstiegskonfiguration für den Beziehungsaufbau und die Herstellung von Vertrauen. Die Interviewanalysen verweisen darauf, dass ein im Gespräch explizit geäusserter oder durch Handeln, Haltung und/oder Emotionen gezeigter Vertrauensvorschuss der Klientel in die SPFFachkräfte (der sich auch in den Gesprächsanalysen zeigt) deren Vertrauensaufbau begünstigen kann und es zu einer wechselseitigen positiven Verstärkung von personalem Vertrauen kommt. So wird bspw. in Fall 2 deutlich, dass die von der Klientin in den ersten Gesprächen gezeigte Offenheit, Ehrlichkeit und Motivation von der Sozialarbeiterin als Indikatoren für die Vertrauenswürdigkeit der Klientin wahrgenommen werden, womit sie sich in ihrem Vorschussvertrauen in die Klientin bestätigt sieht. Dieses Vertrauen in die Klientin nimmt diese wiederum in der Interaktion wahr. Die Klientin macht das an Indikatoren wie offenem Reden der Fachperson, ihrem (selektiven) Erzählen von privaten Dingen und dem direktem Blickkontakt fest. Die Klientin fühlt sich dadurch bestätigt und gestärkt in ihrem Selbstwertgefühl, wie sich in nachfolgendem Zitat der Jugendlichen von Fall 2 zeigt: „Wenn ich merke die Person vertraut mir (.) fühle ich mich 
wertvoll (-) ich denke dann (.) ich habe etwas an mir (.) dass die Person denkt (-) ja ihr kann ich vertrauen. ${ }^{4}$

Wie noch diskutiert wird, führen aber günstige Ausgangsbedingungen nicht automatisch zum Erfolg, und auch ungünstige Ausgangsbedingungen können zu etwas führen, was die Familie als hilfreich bewertet.

Negative Erfahrungen und Einstellungen der Klientel

Demgegenüber stehen Klientinnen und Klienten mit früheren negativen Erfahrungen, bspw. in Fall 3 und Fall 6, die sich in unterschiedlicher Weise auf den Aufbau von Vertrauen in die Fachkraft der SPF auswirken.

In Fall 6 verfügen beide Elternteile über negative Erfahrungen aus der Inanspruchnahme von Kinder- und Jugendhilfemassnahmen sowie aus Kontakten zur Kindesschutzbehörde. Die Kindsmutter, also die Klientin der SPF, thematisiert in den Interviews, dass Fachkräfte und Behörden sich aus ihrer Perspektive nicht (genügend) an ihren Hilfe- bzw. Wirksamkeitserwartungen orientiert haben. Wesentlich ist hier, dass dort, wo sich diese Klientin nicht genügend mit ihren Anliegen ernstgenommen und von den Fachkräften empathisch unterstützt fühlt, sie diese Hilfen als wenig nützlich codiert und sich verletzt fühlt. Darüber hinaus berichtet die Klientin von Entscheidungen von Fachkräften, die gegen ihren Willen getroffen wurden. Interessanterweise sind es Entscheidungen, von denen die Klientin denkt, dass diese in jenem Moment für ihre Kinder positiv waren. Gleichwohl handelte es sich aus ihrer Perspektive um massive Vertrauensverletzungen.

Die negativen Vorerfahrungen wirken sich jedoch bei beiden Elternteilen unterschiedlich aus.
Beim Kindsvater ist ein generalisiertes Misstrauen gegenüber Fachkräften und der KESB festzustellen und es lässt sich eine nachhaltige und aus seiner Sicht nicht mehr korrigierbare Beschädigung des Vertrauens in die Institutionen des Kindesschutzes erkennen, wie sich auch im folgenden Zitat aus einem Interview mit dem Kindsvater aus Fall 6 zeigt:

"[l]ch bin ich bin schon wieder getriggered jetz jetz (-) sorry, weil ähm (.) Vertrauen natürlich wichtig und so ja aber der Punkt, wo ähm (.) wo die allermeisten nicht kennen, (.)n_gefühlten Punkt wo (.) wenn du gesagt bekommst (-) von von irgendjemandem, du darfst das Kind jetzt nicht mitnehmen, wie damals im Krankenhaus, (.) das ist etwas, das ist ein Erlebnis das kannte ich bis dahin noch nicht //[F1: mhm]// und ab dem Punkt mh Vertrauensverhältnis zu irgendwas oder irgendwem aufzubauen (-) äh grad so in äh Institutionen oder irgend ner Behörde oder so ist //das (-) der Restzweifel wird //IMMER bleiben immer."

Zu einem späteren Zeitpunkt wird er zwar der Fachkraft attestieren, dass sie mit guten Absichten handelt, kann sogar einen Nutzen in ihrer Tätigkeit sehen und öffnet sich ein wenig. Dennoch traut er ihr nicht zu, sich gegenüber dem „System" durchzusetzen und er ist sich immer des latenten Risikos einer Fremdplatzierung der Kinder bewusst.

Im Gegensatz dazu lässt die Klientin trotz der negativen Vorerfahrungen die Möglichkeit einer vertrauensvollen Beziehung zur SPF-Fachkraft zu Beginn noch offen. Sie erhofft sich eine Stärkung ihrer Erziehungskompetenzen und eine einseitig parteiliche Unterstützung im Konflikt mit ihrem Ehemann. Solche Hinweise auf einen Vertrauensvorschuss von Seiten der Klientin werden auch im Erstgespräch deutlich, bspw. indem sie

\footnotetext{
${ }^{4}$ Die Interviews wurden in schweizerdeutscher Sprache geführt und transkribiert. Für die Veröffentlichung wurden sie sprachlich dem hochdeutschen angenähert. Folgende Verschriftungsregeln sind zu beachten: 
sich hinsichtlich ihrer Anliegen öffnet. Die Fachkraft nimmt den Paarkonflikt wahr, sieht sich aber für dessen Bearbeitung nicht zuständig und fokussiert ihre Tätigkeit auf die (unerfüllten) Bedürfnisse der Kinder. Es ist dann aber gerade die von der Kindsmutter erhoffte und nicht erbrachte einseitige Parteilichkeit der Fachkraft, die zum Vertrauensverlust führt. Sie fühlt sich zu wenig verstanden und unterstützt sowie in ihren bisherigen negativen Erfahrungen bestätigt, worauf wir mit Blick auf das Beziehungsgeschehen zwischen der Klientin und der Fachkraft später nochmals eingehen.

Zuvor führt diese Enttäuschung der Klientin zu einer weiteren vertrauensrelevanten Einstiegskonfiguration, nämlich den Anliegen, Interessen und Erwartungen der Klientel bezüglich der Hilfe der SPF.

\subsection{Anliegen, Interessen und Erwartungen der Klientel bezüglich der Hilfe}

Für die Entwicklung von interpersonalem Vertrauen ist von Bedeutung, inwiefern bei der Klientel im Ausgangspunkt der Fallarbeit ein intrinsisches Interesse an der Hilfe vorliegt. Gemäss den Analysen geht es aber nicht nur darum, ob die Klientel grundsätzlich an Hilfe interessiert ist. Für die Herstellung von Vertrauen ist relevant, welche Anliegen Klientinnen und Klienten haben sowie von welchen konkreten Erwartungen sie bezüglich der Gestaltung des Hilfesettings ausgehen, bspw. ob zentral mit den Kindern und/oder einem oder zwei Elternteilen gearbeitet werden soll und welche Wirksamkeit sie sich davon versprechen. Diese vertrauensrelevanten Kategorien haben sich zwar mit den bisherigen Ausführungen bereits abgezeichnet und können mit den erwähnten Vorerfahrungen in Verbindung stehen. Dennoch scheint es anaIytisch gewinnbringend, diese gesondert aufzunehmen und auszudifferenzieren. Bezüglich der Frage, warum sich die Klientel auf die SPF einlässt, zeigen sich im Datenmaterial verschiedene Ausgangspunkte, die nun ausführlicher dargestellt werden.
Kein Leidensdruck, kein Unterstützungsbedürfnis aus Perspektive der Klientel

In Fall 3 liegt für die SPF eine Ausgangssituation vor, bei welcher der Fall schon länger prozessiert wird und es durch veränderte Umstände zu einer neuen behördlich angeordneten Massnahme und somit zum Auftrag für die SPF kommt. Die Eltern wünschen sich die Aufhebung der Fremdplatzierung ihrer beiden älteren Kinder. Bei der neuen behördlich angeordneten Massnahme geht es nun einerseits um die Abklärung, ob dem Anliegen der Eltern entsprochen werden kann. Andererseits geht es gemäss Aktenanalysen um die „Überwachung", ob das Wohl des bei den Eltern lebenden neugeborenen Kindes gewährleistet ist. Seitens der Kindesschutzbehörde besteht der Verdacht, dass die Eltern Drogen konsumieren und die Behörde verlangt Haar- bzw. Urinproben von ihnen. Wie die Ergebnisse der Gesprächsanalysen zeigen, interpretiert die SPFFachkraft ihren Auftrag aber weniger als Abklärung, sondern vielmehr als Unterstützung der Eltern bei der Sorge für das Neugeborene, und sie versucht in eine vertrauensvolle Arbeitsbeziehung mit den Eltern zu kommen. Dabei präsentiert sie sich als jemand, die wie eine "Mütterberatungsschwester" oder eine Verwandte Hilfe anbieten kann. ${ }^{5}$ Die Eltern sind äusserst misstrauisch gegenüber der KESB, der Beiständin und auch gegenüber der neuen Massnahme, sehen sich aber durch die wertschätzende Haltung der Fachkraft, ihren Vertrauensvorschuss sowie ihre Bemühungen rund um das Neugeborene nicht bedroht und gewähren so regelmässigen und konfliktfreien Eintritt in die elterliche Wohnung. Dabei präsentieren sie sich als fürsorgliche Eltern, agieren strategisch und verhalten sich vordergründig kooperativ, um ihre Ziele, also die Rückführung der fremdplatzierten Kinder, zu erreichen. Gemäss den Analysen handelt es sich um eine Scheinkooperation.

Kein Leidensdruck, aber ein Unterstützungsbedürfnis aus Perspektive der Klientel

In Fall 5 ist zu Beginn unklar, was die SPF in diesem Fall leisten soll, denn die Klientin präsentiert sich im Erstgespräch kompetent im Umgang mit

\footnotetext{
5 „Mütterberatungsschwestern“ nannte man in der Schweiz bis vor einigen Jahren auf Mütterberatung spezialisierte Krankenschwestern.
} 
alltäglichen Problemen des familiären Zusammenlebens im Allgemeinen und mit ihrem Sohn mit einer autistischen Störung im Besonderen. Die behördlich angeordnete Massnahme kam aus Finanzierungsgründen über eine Gefährdungsmeldung zu Stande. Im ersten Interview ist es der Klientin wichtig zu betonen, dass sie das Hilfesetting nicht als Zwangsmassnahme empfindet, da es auf ihren Wunsch hin eingerichtet wurde. Sie präsentiert sich offen, optimistisch, interessiert und der Hilfe grundsätzlich vertrauend. Gleichwohl ist aber eine gewisse Vorsicht wahrnehmbar. In Kooperation mit der SPF-Fachkraft werden dann eine Reihe von Zielsetzungen im Sinne der Optimierung von ihren Erziehungskompetenzen und der Abläufe im familiären Zusammenleben formuliert, die sich weitgehendst an den Anliegen und Interessen der Klientin orientieren. Die Klientin kann darüber schnell Vertrauen in die Fachkraft aufbauen. Aus ihrer Perspektive wird der Vertrauensaufbau zudem dadurch begünstigt, dass die Fachkraft sich von Beginn an um den Beziehungsaufbau zu den Kindern kümmert und ihr das gut gelingt. Die Fachkraft wiederum sieht viele Ressourcen der Mutter, was ihren Aufbau von Vertrauen begünstigt. ${ }^{6}$ Die Mutter fühlt sich über die ressourcenorientierte Gesprächsführung der Fachkraft in ihren Erziehungskompetenzen bestätigt und gestärkt, nimmt das Vertrauen der Fachkraft in sie wahr und lässt sich auf jene Aspekte ein, die von der Fachkraft als entwicklungs- und verbesserungswürdig herausgearbeitet werden. Gemäss unseren Analysen kommt es zu einem Matching auf der Beziehungsebene, worauf später nochmals gesondert eingegangen wird. Im zweiten Interview formuliert die Klientin nach Beendigung der Tonaufnahme, ,jetzt ist der Zwang weg" als Teil eines wiederholten Fazits zur geglückten Aufnahme der Zusammenarbeit mit der Fachkraft. Sie war sich also doch des Zwangscharakters des Settings bewusst und ein Teil inrer Selbstdarstellung als kompetente Mutter ohne grössere Probleme kann dem Kontext geschuldet sein.

\section{Leidensdruck bei der Klientel}

In Fall 1 hat die Kindsmutter aufgrund von eigenem Interesse und Leidensdruck um Hilfe von der SPF gebeten, wie sich auch in folgendem Interviewausschnitt mit der Kindsmutter zeigt:

"Ich denke also von meiner Seite ist es einfach (.) das ich einfach muss sagen (.) eben eben (.) weil ich selber sehe in welcher dings wir leben (-) $\mathrm{h}$ und ich etwas will ändern (.) // oder [Forscherin:mhm] $\|$ eben wie gesagt ich komm ich machs ja auf freiwilliger Basis und ich will etwas verändern $h$ ich will mich verändern (.) also ins positive, wo es die_eh die Erziehung anbetrifft (.) ich will ehm (.) eine andere Beziehung zu den Kinder bekommen."

Gemäss den Interviews fühlt sie sich aufgrund der schwierigen Trennung vom Kindsvater und im Umgang mit einem ihrer Kinder mit einem Aufmerksamkeitsdefizitsyndrom hoch belastet. Die SPF wurde von einer Therapeutin empfohlen. Mit Einverständnis der Mutter richtete die Therapeutin eine Gefährdungsmeldung an die KESB. Dabei scheinen auch Finanzierungsgründe eine Rolle gespielt zu haben. In Fall 4 und Fall 5 gibt es im Datenmaterial ebenfalls Hinweise darauf, dass die Auslösung einer Gefährdungsmeldung in der einvernehmlichen Absprache mit mindestens einem Elternteil erfolgte und diese einen Unterstützungsbedarf erkennen. Aber auch in Fall 2, der zunächst gegen den Willen der Eltern und der Jugendlichen konstituiert wurde, artikuliert die Jugendliche ein Leiden an den Problemen ihrer Lebensführung. Sie alle verfügen über konkrete Vorstellungen, was aus ihrer Sicht das Problem ist bzw. wer innen ein Problem macht (z. B. der Partner oder die Partnerin; die Lehrpersonen des Kindes oder Mitschülerinnen und Mitschüler, die finanzielle Situation, eine Erkrankung des Kindes), und erhoffen sich in Bezug darauf Unterstützung durch die SPF.

Sie verfügen auch über klare Vorstellungen im Hinblick auf die Problembearbeitung im Sinne von Erwartungen bezüglich mit wem die Fachkraft innerhalb der Familie an welchen Themen

\footnotetext{
${ }^{6}$ Fall 6 wird dann zeigen, dass das Vorhandensein vieler Ressourcen unter Umständen nicht ausreicht, um das Kindeswohl zu sichern.
} 
zu arbeiten hat. Dieser Leidensdruck und das daraus resultierende Interesse an der Hilfe stellt für den Aufbau von Vertrauen eine sehr günstige Voraussetzung dar. Dennoch führt sie gemäss den Analysen im weiteren Verlauf nicht automatisch zum Erfolg, wie sich im Fall 1 zeigt. Es können auf der Sach- wie auch der Beziehungsebene Spannungsfelder entstehen, welche an zwei weitere vertrauensrelevante Einstiegskonfigurationen gekoppelt sind, nämlich an den „Risikofaktor Kindeswohlgefährdung“ (Abschnitt 4.3) und das Matching auf der Beziehungsebene (Abschnitt 4.4).

\subsection{Risikofaktor Kindeswohlgefährdung}

Für den weiteren Aufbau und die Stärkung von personalem Vertrauen ist es günstig, wenn die Anliegen der Fachkräfte und ihre Tätigkeiten möglichst nah bei den zuvor erwähnten Interessen, Anliegen und Erwartungen der Eltern liegen. In jenen Fällen, wo die Fachkräfte auf der Basis ihrer Expertise zu einer anderen Problemdeutung als die Eltern gelangen und zugunsten der Stärkung des Kindeswohls Interventionen ergreifen, die nicht mehr dem ursprünglichen Anliegen der Eltern entsprechen, kommt es zu vertrauensrelevanten Spannungen. Hier sind die Fachkräfte gefordert, die Bedürfnisse und Anliegen der Eltern sowie ihre Interventionen zur Stärkung des Kindeswohls möglichst gut miteinander in Einklang zu bringen. Je nach Fallproblematik lässt sich das aber sachlich-inhaltlich schwer vereinbaren. Dort, wo dieser Balanceakt nicht gelingt bzw. nicht gelingen kann, verlieren Eltern gemäss unseren Analysen das entstandene Vertrauen in die SPF-Fachkraft.

In dem zuvor erwähnten Fall 1, bei dem die Kindsmutter von sich aus Hilfe angefordert hat, kann sich das ursprünglich günstige Vertrauenspotential nicht entwickeln. Nebst dem Aspekt, dass ohne Begründung nach einem gelungenen Start unerwartet die zugeteilte Fachkraft ausgewechselt wird und es zu einer ersten Vertrauensenttäuschung kommt, gelingt es der neuen Fachkraft aus Sicht der Kindsmutter weniger gut, zu ihren Kindern in Beziehung zu treten. Zweifel an deren Fachkompetenz sind ebenfalls ungünstig für die Herstellung von Vertrauen. Zudem äussert die Fachkraft gegenüber der Mutter, dass sie das Kindeswohl gefährdet sieht, was für die
Kindsmutter unerwartet kommt. Sie verliert daraufhin gänzlich das Vertrauen in die Fachperson. Wie die Analysen zeigen, gibt sie sich aber weiterhin kooperativ und versucht darüber, die Gefahr der Fremdplatzierung des Kindes zu minimieren. Im Indikationsbericht wird dann die Hilfsakzeptanz der Mutter auch als entscheidender Grund für den Verbleib des Kindes bei der Mutter genannt. Interessanterweise äussert auch die Fachkraft in den Interviews, dass sie der Klientin nur noch bedingt vertraut, aber auch sie nimmt an der Inszenierung einer Scheinkooperation teil. Die Beziehung verbleibt auf der Ebene von Verlässlichkeit, d. h. es entwickelt sich eine Vorstellung dessen, was vom Gegenüber zu erwarten ist und wo die Grenzen und Gefahren der Beziehung liegen. Innerhalb dieser Grenzen funktioniert die Kooperation in Fall 1 gut und erreicht auch gewisse Ziele. Heikle Punkte werden jedoch gemieden und Hoffnungen in die Hilfeleistung werden von beiden Seiten zurückgefahren. Gegen aussen neigen aber sowohl die Fachperson als auch die Klientin dazu, den Verlauf positiver darzustellen, als er sich in unseren Analysen zeigt.

\subsection{Das Matching auf der Beziehungsebene als Schlüsselfaktor für den Vertrauensaufbau}

In verschiedenen Fällen, v. a. in Fall 2, 5 und 6, wird deutlich, dass die Klientel nicht nur ein konkretes Anliegen in Bezug auf die familiäre Lebensführung, sondern aus ihrem biographischen Geworden-Sein und auch aus der aktuellen Lebenssituation heraus einen die Beziehungsebene adressierenden Mangel bzw. eine Bedürftigkeit in die Arbeitsbeziehung miteinbringen. Das zeigt sich in den Interviews wie aber auch in den untersuchten Gesprächen bspw. in einem ausgeprägten Bedürfnis nach Anerkennung durch die Fachperson, dem Wunsch nach einseitiger Parteilichkeit, jemanden zu haben, der zuhört und Interesse zeigt, sich Zeit nimmt, nach uneingeschränkter Verfügbarkeit der Fachkraft, nach einer VertretungNermittlung der eigenen Anliegen gegenüber Dritten bis hin zum Bedarf eines Vorbildes für persönliche Entwicklungsprozesse. 
Ein Teil dessen kommt auch in folgendem Interviewausschnitt mit der Jugendlichen (Fall 2) zum Ausdruck:

„Sie [die Fachkraft] hört zu und wenn ich mal etwas nicht verstehe und nachfrage (.) dann sagt sie es gerade (-) sie erklärt sehr gut (-) erklärt was ich genau genau gefragt habe (-) kann Antwort geben (.) die ich verstehe (.) sie nimmt sich Zeit für mich (.) sagt nicht oh es sind zwei Stunden rum (.) der Termin ist fertig (-) sie fragt immer am Schluss ob ich noch Fragen habe (...) sie erzählt, dass sie alles fragen kann (...) sie kann es super gut erklären bis ins Detail rein was ich genau machen muss [Lehrstellensuche] und sie hilft mir mit meinem Hobby (.) bei dem ich gehen will (-) sie ist wie ein wenig meine beste Kollegin (.) einfach dass sie etwas älter ist (lacht) und beisteht egal wo ich gehe und egal was ich habe (...) sie hilft mir und sie ist auch recht nett (-) es gibt ja auch Jugendcoach die so ein wenig beim Beruf bleiben (.) aber sie fragt auch Sachen wie waren Ferien (.) Hochzeit (-) fragte mich nach Bildern (-) zeigt Interesse in mich rein (-) will wissen wer bin ich wie bin ich."

Zudem ergeben sich im Datenmaterial bei den Klientinnen und Klienten Hinweise auf ihre charakteristischen Muster der Beziehungsgestaltung, bspw. in dem sie sich gegenüber der Fachkraft eher als hilfsbedürftig präsentieren und eine Art mütterliche Unterstützung oder empathische verlässliche Freundin suchen, oder aber starken Wert darauflegen, gerade nicht als hilfsbedürftig, sondern auf Augenhöhe und als kompetent adressiert zu werden. Demgegenüber stehen die Fachkräfte, die sich auch auf ihre spezifische Weise in diesen Aufbau von Vertrauen und der Arbeitsbeziehung einbringen. Sie agieren nicht nur rollenförmig, sondern werden im Datenmaterial als ganze Menschen mit ihrer Persönlichkeit mehr oder weniger sichtbar. Wie in Fall 2 arbeiten manche Fachkräfte sogar selektiv und bewusst damit.

In Fall 2 und Fall 5 bilden nun das Beziehungsangebot sowie der persönliche Arbeitsstil der SPF-Fachkraft das perfekte Gegenstück dessen, was die Klientel bewusst oder unbewusst nicht nur inhaltlich, sondern auch auf der Beziehungsebene sucht. Es geht hier also um eine Passung in der Interaktion, um das Matching auf der Beziehungsebene zwischen den Klientinnen bzw. Klienten und der SPF-Fachkraft, das über Aspekte wie Sympathie, Alter, Aussehen und Geschlecht hinausgeht. In Fall 2 und Fall 5 ist es gerade dieses individuelle Matching, welches ein Schlüsselfaktor für den gelungenen interpersonellen Vertrauensaufbau darstellt.

Die Fachkräfte aus Fall 2 und 5, bei denen es zu einem solchen Matching kommt, agieren grundsätzlich günstig im Beziehungsaufbau, was sich bspw. in der Art ihrer wertschätzenden und am Gegenüber interessierten Gesprächsführung zeigt. Es wäre aber verkürzt anzunehmen, dass diese Kompetenz für den Vertrauensaufbau alleinig ausreichend ist. In der Analyse des Falles 6, an dem dieselbe Fachperson wie in Fall 5 beteiligt ist, zeigt sich von Beginn an, dass es mit der Klientin von Fall 6 nicht zu dieser Passung kommt. Die Klientin von Fall 6 zeigt sich wie oben schon ausgeführt zwar offen für den Aufbau einer Arbeitsbeziehung und wünscht sich gegenseitiges Vertrauen. Sie bringt jedoch eine für den Vertrauensaufbau folgenreiche Disposition an Beziehungsmuster in diese Arbeitsbeziehung mit ein, die nach Aufmerksamkeit, emphatischer Zuwendung, Verstehen ihrer Problemsicht und die Einnahme einer parteilichen Haltung für ihre Anliegen sucht. Zudem liegen bei der Klientin Persönlichkeitseigenschaften vor, welche die Ausbildung von Vertrauen erschweren, so z. B. eine besondere Sensibilität für die Handlungen des Gegenübers, die sie als negative Beziehungsbotschaften ausgelegt, eine strukturelle Unsicherheit, die sie aber in der Interaktion verdeckt und eine mangelnde Fähigkeit zur Empathie. Der Arbeitsstil dieser Fachperson, der von der Klientin in Fall 5 gerade auch wegen dem klaren und etwas konfrontativ-fordernden sowie gleichzeitig empathischen und wertschätzenden Gesprächsstil gesucht und geschätzt wird, löst bei der Klientin von Fall 6 eine andere Reaktion aus. Hier führt dieser Arbeitsstil sowie die Nichteinnahme der von der Klientin erhofften einseitigen Parteilichkeit dazu, dass die Klientin verletzt reagiert, sich nicht verstanden fühlt und die Fachkraft und ihre Hilfe als nicht wirksam abwertet.

Gerade die sehr gelungenen Fälle mit dem Matching machen deutlich, dass sozialpädagogische Familienhilfe eine Arbeit ist, die sich in und aus 
der Beziehung realisiert. Für einen günstigen Vertrauensaufbau bedarf es der Passung zwischen Zielen, Interessen und Erwartungen auf der Sachebene, der Passung auf der Beziehungsebene und über eine Hilfe, die von den Betroffenen als hilfreich erachtet wird.

\section{ZUSAMMENFASSUNG UND DISKUSSION}

Unsere empirischen Analysen zeigen, dass die Annahmen von Oevermann (2009) und Schütze (1992) über die Bedeutung von Vertrauen in Arbeitsbeziehungen von Professionsangehörigen und Klientinnen bzw. Klienten auch in sozialpädagogischen Familienbegleitungen im Zwangskontext Geltung besitzen. Vertrauen wird als ein konstitutives Element dieser Arbeitsbeziehung sowohl von Fachkräften als auch Klientinnen und Klienten angesehen und angestrebt, sofern eine solche Arbeitsbeziehung überhaupt erwünscht ist. Die theoretische Konzeptualisierung und die darin enthaltenen Differenzierungen des Begriffs im interdisziplinären Fachdiskurs um Vertrauen, an die wir anschliessen, erwiesen sich empirisch als fruchtbar. Vertrauen hat sich in unseren AnaIysen als ein Phänomen gezeigt, das sich als generalisiertes Vertrauen auf abstrakte Institutionen des Kindesschutzes bezieht (Luhmann 1968; Giddens 1990) sowie auf die Fachkräfte von Berufen, die in diesem System tätig sind. Davon unterscheiden lässt sich das Vertrauen, welches konkreten Personen im Verlaufe einer Massnahme entgegengebracht wird. Sobald die Massnahme begonnen hat, treffen Klientinnen bzw. Klienten auf Fachkräfte, die im Sinne von Giddens (1990) zugleich systemvertretende Personen als auch konkrete Personen mit spezifischen, für die Vertrauensbildung relevanten Merkmalen sind. Für die Vertrauensbildung ist bedeutsam, wie die Personen ihre Aufgabe ausüben. Dabei ist nicht nur die fachspezifische Expertise wichtig, sondern auch persönliche Eigenschaften. Die im Fachdiskurs der Sozialen Arbeit kontrovers diskutierte Frage, ob Vertrauen sich auf Fachkräfte als Rollenausübende oder als konkrete Personen bezieht (Wagenblass 2004; Arnold 2009; Zeller 2012), lässt sich nach unseren Ergebnissen in beiden Fällen bejahen. Die Vertrauensbildung ist gemäss unseren Analysen ein reziproker und dynamischer Prozess. Beide Seiten, Fachkräfte und Klientin- nen bzw. Klienten, investieren in die Vertrauensbildung, leisten also im Sinne Luhmanns (1968) einen Vertrauensvorschuss. Wechselseitige Verstärkungen der Vertrauensbildung sind bei günstigen Voraussetzungen zu beobachten. Die Vertrauensbildung zeigt sich als voraussetzungs- und anspruchsvoller Prozess, der sich in seiner Dynamik und seinem Ergebnis von der Eingangskonstellation her nicht sicher prognostizieren lässt.

Als Bedingungen für die Vertrauensbildung auf Seiten der Klientinnen und Klienten spielen zu Beginn der Massnahmen Vorerfahrungen mit Fachkräften und Institutionen des Kindesschutzsystems sowie Persönlichkeitsdispositionen und Leidensdruck eine wichtige Rolle. Im Prozess hängt die Vertrauensbildung dann davon ab, inwieweit die Person und das Vorgehen der Fachkräfte den Interessen und Erwartungen der Klientinnen und Klienten entspricht (zur „Passung" als wirksamem Faktor für den Hilfeprozess siehe auch Fröhlich-Gildhoff 2003; 2014). Es bedarf der Passung zwischen Zielen, Interessen und Erwartungen auf der Sachebene wie auch auf der Interaktionsebene der Arbeitsbeziehung und über eine Hilfe, die von den Betroffenen als hilfreich erachtet wird. Eine grosse Schwierigkeit (Brauchli 2021) kann darin bestehen, dass die Erfüllung von Interessen und Erwartungen der Eltern sich nicht mit der Gesamtausrichtung der Massnahme an der Förderung des Kindeswohls decken. Wenn sich im Zuge von Massnahmen das Risiko einer Intervention zur Abwendung einer Kindeswohlgefährdung konkretisiert, kann dies zu einem Vertrauensverlust auf Seiten der Klientinnen bzw. Klienten führen. Erschwerend für die Vertrauensbildung ist auch, dass die Klientinnen und Klienten teilweise und gerade bei konflikthaften Paarbeziehungen dazu tendieren, von den Fachkräften eine Parteinahme für ihre Position sowie die Anerkennung ihrer Person und ihrer Erziehungskompetenz zu erwarten, die die Fachkräfte aus sachlichen Gründen nicht leisten können, z. B. weil eine einseitige Parteinahme für ein Elternteil die Kooperation mit dem anderen verunmöglicht oder weil die Fachkräfte bezüglich der Erziehungskompetenz Defizite sehen, die die betreffenden Elternteile nicht anerkennen. Diese Erfolgsbedingungen und Schwierigkeiten der Vertrauensbildung rund um das Thema Passungsverhältnis bzw. Matching von Interessen 
und Erwartungen von Klientinnen und Klienten auf der einen Seite und der Person sowie dem Vorgehen der Fachkraft auf der anderen Seite hat sich als anspruchsvoller herausgestellt, als wir das vor unseren Analysen erwartet hätten. Die Vertrauensbildung ist sehr stark von einer Arbeitsweise abhängig, die sich auf die Interessen und Erwartungen von Klientinnen und Klienten einlassen kann, wobei aber den Fachkräften bedingt durch ihren Auftrag Grenzen gesetzt sind. Unseres Erachtens wäre es lohnenswert, diesem Thema im Hinblick auf die Indikationsstellung für SPF-Massnahmen in einer weiteren Forschungsarbeit genauer nachzugehen.

Zudem würde es sich lohnen, Institutionalisierungen und Massnahmen des Kindesschutzsystems im Hinblick auf die möglichst optimale Förderung von Vertrauensbildungsprozessen zu überdenken. Verbesserungsmöglichkeiten bestehen unserer Meinung nach in der Transparenz darüber, wer mit welcher Begründung die Verantwortung für getroffene Entscheidungen trägt, in Bezug auf Wahlmöglichkeiten der Klientinnen und Klienten hinsichtlich der Personen, die mit innen zusammenarbeiten, in Bezug auf u. a. finanzielle Sachzwänge, die dazu führen, dass Massnahmen unnötigerweise durch Verfügung der Kindesschutzbehörden und damit im Zwangskontext aufgegleist werden, und nicht zuletzt auch im Hinblick auf die Beendigung der Massnahme, wenn kein Vertrauen aufgebaut werden konnte oder dieses verloren gegangen ist. Vertrauen stellt eine unabdingbare Voraussetzung für den Erfolg einer Hilfemassnahme dar. Auch wenn es in erster Linie um Kontrolle des Kindeswohls geht, ist ein Mindestmass an Vertrauen nötig (vgl. Klatetzki 2019, S. 184).

\section{LITERATUR}

Arnold, Susan (2009): Vertrauen als Konstrukt. Sozialarbeiter und Klient in Beziehung. Marburg: Tectum Verlag.

Baier, Annette (1986): Trust and Antitrust. In: Ethics, 96 (2), 231-260.

Baier, Annette (1991): Trust and Its Vulnarabilites \& Sustaining Trust. In: Tanner Lectures on Human Values. Salt Lake City: University of Utah Press, 109-174.
Brauchli, Simone (2021): Das Wohl der Kinder und die Selbstbestimmung der Eltern. Eine qualitative Untersuchung zur Sozialpädagogischen Familienbegleitung in der Schweiz. Weinheim: Beltz Juventa.

Cleppien, Georg (2012): Über die Schwierigkeit Klient/innen zu vertrauen. In: Tiefel, Sandra/Zeller, Maren (Hrsg.): Vertrauensprozesse in der Sozialen Arbeit. Baltmannsweiler: Schneider-Verlag Hohengehren, 49-66.

Cook, Karen S./Hardin, Russell/Levi, Margaret (2005): Cooperation Without Trust? New York: Russell Sage Foundation.

Cossar, Jeanette/Brandon, Marian/Jordan, Peter (2016): 'You've got to trust her and she's got to trust you': Children's views on participation in the child protection system. In: Child \& Family Social Work, 21 (1), 103-112.

Davies, Hayley (2019): Trust and Distrust: Listening to Children about Their Relationships with Professionals. In: Social Sciences, 8 (9), 251.

Dumbrill, Gary C. (2006): Parental experience of child protection intervention: A qualitative study. In: Child Abuse \& Neglect, 30 (1), 27-37.

Franzheld, Tobias (2017): ,Verdacht' als theoretische Reflexion und analytische Konzeption der Kinderschutzforschung. In: Sozialer Sinn, 18 (2), 103.

Freres, Katharina (2020): Verdachtsabkärung im Kinderschutz: Ambivalenzen des Fallverstehens. In: Kelle, Helga/Dahmen, Stephan (Hrsg.): Ambivalenzen des Kinderschutzes. Weinheim/Basel: Beltz, 42-61.

Fröhlich-Gildhoff, Klaus (2003): Einzelbetreuung in der Jugendhilfe: Konzepte, Prozesse und wirksame Faktoren. Münster: Lit Verlag.

Fröhlich-Gildhoff, Klaus (2014): § 31 SGB VIII: Sozialpädagogische Familienhilfe. In: Macenaere, Michael/Esser, Klaus/Knab, Eckhart/Hiller, Stephan (Hrsg.): Handbuch der Hilfen zur Erziehung. Freiburg im Breisgau:Lambertus-Verlag, 110115.

Gibson, Matthew (2020): The shame and shaming of parents in the child protection process: Findings from a case study of an English child protection service. In: Families, Relationships and Societies, 9 (2), 217-233. 
Giddens, Anthony (1990): The Consequences of Modernity. Stanford: Stanford University Press.

Goffman, Erving (1959): The Presentation of Self in Everyday Life. New York: Anchor Books.

Hall, Christopher/Mäkitalo, Åsa/Slembrouck, Stef/Doherty, Paula (2013): Pursuing Trust in Child Protection Meetings: Familiarization and Informality. In: Candlin, Christopher N. /Crichton, Jonathan (Hrsg.): Discourses of Trust. Basingstoke: Palgrave Macmillan, 100-118.

Hansjürgens, Rita (2018): In Kontakt kommen. Analyse der Entsteheung einer Arbeitsbeziehung in Suchtberatungsstellen. Baden-Baden: Tectum.

Hardin, Russell (2002): Trust and Trustworthiness. New York: Russell Sage Foundation.

Husby, Inger Sofie Dahlø/Sletteb $\varnothing$, Tor/Juul, Randi (2018): Partnerships with children in child welfare: The importance of trust and pedagogical support. In: Child \& Family Social Work, 23 (3), 443-450.

Klatetzki, Thomas (2019): Narrative Praktiken: Die Bearbeitung sozialer Probleme in den Organisationen der Kinder- und Jugendhilfe. Weinheim/Basel: Beltz Juventa.

Lefevre, Michelle/Hickle, Kristine/Luckock, Barry/Ruch, Gillian (2017): Building Trust with Children and Young People at Risk of Child Sexual Exploitation: The Professional Challenge. In: The British Journal of Social Work, 47 (8), 24562473.

Lewis, J. David/Weigert, Andrew J. (1985a): Social Atomism, Holism, and Trust. In: The Sociological Quarterly, 26 (4), 455-471.

Lewis, J. David/Weigert, Andrew J. (1985b): Trust as a Social Reality. In: Social Forces, 63 (4), 967-985.

Luhmann, Niklas (1968): Vertrauen. Ein Mechanismus der Reduktion sozialer Komplexität. Stuttgart: Ferdinand Enke.

McLeod, Carolyn (2015): Trust. In: Zalta, Edward N. (Hrsg.): The Stanford Encyclopedia of Philosophy. Fall 2015 Edition. Stanford: Stanford University.

Möllering, Guido (2001): The Nature of Trust: From Georg Simmel to a Theory of Expectation,
Interpretation and Suspension. In: Sociology, 35 (2), 403-420.

Möllering, Guido (2005): The Trust/Control Duality: An Integrative Perspective on Positive Expectations of Others. In: International Sociology, 20 (3), 283-305.

Möllering, Guido (2006): Trust: Reason, Routine, Reflexivity. Oxford/Amsterdam: Elsevier.

Möllering, Guido (2013): Process Views of Trusting and Crises. In: Bachmann, Reinhard/Zaheer, Akbar (Hrsg.): Handbook of Advances in Trust Research. Cheltenham: Edward Elgar, S. 285306.

Oevermann, Ulrich (2000): Die Methode der Fallrekonstruktion in der Grundlagenforschung sowie der klinischen und pädagogischen Praxis. In: Kraimer, Klaus (Hrsg.): Die Fallrekonstruktion. Sinnverstehen in der sozialwissenschaftlichen Forschung. Frankfurt a. M.: Suhrkamp, 58-156.

Oevermann, Ulrich (2009): Die Problematik der Strukturlogik des Arbeitsbündnisses und der Dynamik von Übertragung und Gegenübertragung in einer professionalisierten Praxis von Sozialarbeit. In: Becker-Lenz, Roland/Busse, Stefan/Ehlert, Gudrun/Müller, Silke (Hrsg.): Professionalität in der Sozialen Arbeit. Standpunkte, Kontroversen, Perspektiven. Wiesbaden: VS Verlag für Sozialwissenschaften, 113-142.

Richter, Martina (2013): Die Sichtbarmachung des Familialen. Gesprächspraktiken in der Sozialpädagogischen Familienhilfe. Weinheim: Beltz Juventa.

Rüegger, Cornelia (2021): Fallkonstitution in Gesprächen Sozialer Arbeit. Prozesse und Praktiken der organisationalen und interaktiven Produktion des Falles. Wiesbaden: Springer.

Schütze, Fritz (1992): Sozialarbeit als „bescheidene Profession". In: Dewe, Bernd/Ferchhoff, Wilfried/Radtke, Frank-Olaf (Hrsg.): Erziehen als Profession. Wiesbaden: Springer Fachmedien, 132-170.

Tiefel, Sandra (2012): Strategien der Vertrauensherstellung im Beratungsprozess. In: Tiefel, Sandra/Zeller, Maren (Hrsg.): Vertrauensprozesse in der Sozialen Arbeit. Baltmannsweiler: Schneider-Verlag Hohengehren, 15-32. 
Wagenblass, Sabine (2004): Vertrauen in der Sozialen Arbeit. Theoretische und empirische Ergebnisse zur Relevanz von Vertrauen als eigenständiger Dimension. Weinheim: Juventa.

Zeller, Maren (2012): Persönliches vs. spezifisches Vertrauen. Ein Spannungsfeld professionellen Handelns in den Erziehungshilfen. In: prozesse in der Sozialen Arbeit. Baltmannsweiler: Schneider-Verlag Hohengehren, 93-105. 
Tabelle 1 Eckdaten Fallkonstellationen Sample

\begin{tabular}{|c|c|c|c|c|}
\hline $\begin{array}{l}\text { Fall } \\
\#\end{array}$ & $\begin{array}{l}\text { Familienkonstellation (unge- } \\
\text { fähres Alter der Kinder zu Be- } \\
\text { ginn der Massnahme) }\end{array}$ & $\begin{array}{l}\text { Arbeitsbezie- } \\
\text { hungen SPF } \\
\text { mit Familien- } \\
\text { mitgliedern }\end{array}$ & $\begin{array}{l}\text { Form des Zwangs durch } \\
\text { SPF }\end{array}$ & $\begin{array}{l}\text { Interview- } \\
\text { partner/-in in } \\
\text { Familie }\end{array}$ \\
\hline 1 & $\begin{array}{l}\text { Aus erster Ehe der Mutter } \\
\text { Sohn (18 J.) und Tochter (21 J.), } \\
\text { aus zweiter Ehe Sohn (8 J.) und } \\
\text { Tochter (14 J.), Mutter und } \\
\text { (Stief-)Vater in Trennung, zu } \\
\text { Beginn noch im gemeinsamen } \\
\text { Haushalt lebend }\end{array}$ & $\begin{array}{l}\text { Primär mit } \\
\text { Mutter, sekun- } \\
\text { där mit dem } \\
\text { Ehemann }\end{array}$ & $\begin{array}{l}\text { KESB-Massnahme (Mutter } \\
\text { erklärt sich einverstanden } \\
\text { mit dem Einreichen einer } \\
\text { Gefährdungsmeldung durch } \\
\text { einen kinderpsychiatrischen } \\
\text { Dienst zwecks Finanzierung } \\
\text { der Massnahme) }\end{array}$ & Mutter \\
\hline 2 & $\begin{array}{l}\text { Tochter (15 J.), Sohn (19 J.), } \\
\text { Mutter. Der Vater lebt auf- } \\
\text { grund einer Ausschaffung im } \\
\text { Herkunftsland. }\end{array}$ & $\begin{array}{l}\text { Primär mit } \\
\text { Tochter, punk- } \\
\text { tuell mit Mutter }\end{array}$ & $\begin{array}{l}\text { KESB-Massnahme (Jugend- } \\
\text { coaching) }\end{array}$ & Jugendliche \\
\hline 3 & $\begin{array}{l}\text { Zwei Töchter ( } 9 \text { J.; } 5 \text { J.), fremd- } \\
\text { platziert und einen } \\
\text { Sohn (Baby) bei den Eltern le- } \\
\text { bend, } \\
\text { Mutter, Vater }\end{array}$ & $\begin{array}{l}\text { Mit Mutter und } \\
\text { Vater }\end{array}$ & $\begin{array}{l}\text { KESB-Massnahme (Abklä- } \\
\text { rung ob Rückplatzierung } \\
\text { der Töchter wieder möglich } \\
\text { und Überwachung des } \\
\text { Neugeborenen) }\end{array}$ & $\begin{array}{l}\text { Mutter und } \\
\text { Vater (zwei- } \\
\text { tes Interview } \\
\text { nur mit Mut- } \\
\text { ter) }\end{array}$ \\
\hline 4 & $\begin{array}{l}\text { Sohn aus erster Ehe des Va- } \\
\text { ters (13 J.), Sohn aus zweiter } \\
\text { Ehe (4 J.) sowie Tochter (1 J.), } \\
\text { (Stief-)Mutter, Vater }\end{array}$ & $\begin{array}{l}\text { Zunächst und } \\
\text { primär mit } \\
\text { Sohn (13 J.), } \\
\text { später und se- } \\
\text { kundär auch } \\
\text { mit Stiefmutter } \\
\text { und Vater }\end{array}$ & $\begin{array}{l}\text { Kein behördlicher Zwang; } \\
\text { Beteiligung der Mutter und } \\
\text { des Sohnes aufgrund des } \\
\text { Wunsches des Ehemannes } \\
\text { bzw. Vaters }\end{array}$ & $\begin{array}{l}\text { Vater, Stief- } \\
\text { mutter, Sohn }\end{array}$ \\
\hline 5 & $\begin{array}{l}\text { Tochter (4 J.) und zwei Söhne } \\
\text { (15 J., } 12 \text { J.) aus einer Bezie- } \\
\text { hung mit einem anderen Mann } \\
\text { Mutter, alleinerziehend }\end{array}$ & $\begin{array}{l}\text { Primär mit } \\
\text { Mutter und den } \\
\text { beiden jünge- } \\
\text { ren Kindern }\end{array}$ & $\begin{array}{l}\text { KESB Massnahme } \\
\text { (Mutter erklärt sich einver- } \\
\text { standen mit dem Einreichen } \\
\text { einer Gefährdungsmeldung } \\
\text { durch eine Kindertherapeu- } \\
\text { tin zwecks Finanzierung der } \\
\text { Massnahme) }\end{array}$ & Mutter \\
\hline 6 & $\begin{array}{l}\text { Zwei Töchter (9 M. und knapp } \\
3 \text { J.), Mutter, Vater }\end{array}$ & $\begin{array}{l}\text { Primär mit } \\
\text { Mutter und Va- } \\
\text { ter (einzeln, ge- } \\
\text { meinsam), } \\
\text { punktuell Inter- } \\
\text { aktionen mit } \\
\text { Kindern }\end{array}$ & KESB-Massnahme & Mutter \\
\hline
\end{tabular}




\section{Zu den Autor*innen}

Cornelia Rüegger ist tätig als wissenschaftliche Mitarbeiterin am Institut für Professionsforschung und entwicklung an der Hochschule für Soziale Arbeit der FHNW. Ihre Schwerpunkte sind die Professionsund Gesprächsforschung in der Sozialen Arbeit mit Fokus auf die Ausgestaltung sozialer Diagnostik/Fallkonstitution und Prozessgestaltung in unterschiedlichen Arbeitsfeldern (Soziale Arbeit im Gesundheitswesen; Kinder- und Jugendhilfe, Kindes- und Erwachsenenschutz).

Joel Gautschi ist Dozent an der ZHAW Zürcher Hochschule für Angewandte Wissenschaften. Er forscht zu Kinderschutz, Urteils- und Entscheidungsfindung und Vertrauen.

Roland Becker-Lenz ist Dozent an der Hochschule für Soziale Arbeit der FHNW. Seine Arbeitsschwerpunkte sind die Professions- und Bildungsforschung im Bereich der Sozialen Arbeit.

Fabienne Rotzetter ist wissenschaftliche Mitarbeiterin am Institut für Professionsforschung und -entwicklung an der HSA FHNW. Ihr Schwerpunkt ist die Professionsforschung in der Sozialen Arbeit mit Fokus auf Fragen der Beziehungsgestaltung, der Diagnostik und der Verbindung von Theorie und Praxis.

\section{Kontakt}

Dr. Cornelia Rüegger

Hochschule für Soziale Arbeit FHNW

Institut für Professionsforschung und -entwicklung

Riggenbachstrasse 16

$\mathrm{CH}-4600$ Olten

E-Mail: cornelia.rueegger@fhnw.ch

URL: https://www.fhnw.ch/de/personen/cornelia-rueegger

Dr. Joel Gautschi

ZHAW Soziale Arbeit

Institut für Kindheit, Jugend und Familie

Pfingstweidstrasse 96

$\mathrm{CH}-8037$ Zürich

E-Mail:joel.gautschi@zhaw.ch

URL: https://www.zhaw.ch/de/ueber-uns/person/gaud/

Prof. Dr. Roland Becker-Lenz

Hochschule für Soziale Arbeit FHNW

Institut für Professionsforschung und -entwicklung

Riggenbachstrasse 16

$\mathrm{CH}-4600$ Olten

E-Mail: roland.becker@fhnw.ch

URL: https://www.fhnw.ch/de/personen/roland-becker-lenz

Fabienne Rotzetter

Hochschule für Soziale Arbeit FHNW

Institut für Professionsforschung und -entwicklung

Riggenbachstrasse 16

$\mathrm{CH}-4600$ Olten

E-Mail: fabienne.rotzetter@fhnw.ch

URL: https://www.fhnw.ch/de/personen/fabienne-rotzetter 